\title{
ON THE AREA DISTORTION BY QUASICONFORMAL MAPPINGS
}

\author{
A. EREMENKO AND D. H. HAMILTON \\ (Communicated by Albert Baernstein II)
}

\begin{abstract}
We give the sharp constants in the area distortion inequality for quasiconformal mappings in the plane.
\end{abstract}

Astala [1] proved the following theorem conjectured by Gehring and Reich in [3]:

Theorem A. Let $f$ be a $K$-quasiconformal mapping of $D=\{z:|z|<1\}$ onto itself with $f(0)=0$. Then for any measurable $E \subset D$ we have

$$
|f(E)| \leq C(K)|E|^{1 / K}
$$

where $|\cdot|$ stands for the area.

The first author [2] obtained a shorter proof which did not make use of the elaborate Thermodynamic Formalism and Holomorphic Motion Theory of the original proof of Astala. In late 1992 the second author [4] circulated a minimal proof which gives sharp bounds for the constants under the normalization $f \in$ $\Sigma(K)$, i.e. $f$ is a $K$-quasiconformal mapping of the plane which is conformal on $C \backslash \bar{D}$ and $f(z)=z+o(1)$ near $\infty$. In the interests of having a short sharp proof we combined our efforts.

Usually in what follows $\Delta$ is the closed unit disk $\{z:|z| \leq 1\}$, but any compact set of transfinite diameter 1 will do (and this is important in our proof). We note that this normalization implies that for any $E \subset \Delta$ the area of $E$ and $f(E)$ is bounded by $\pi$.

Theorem 1. Let $f$ be a $K$-quasiconformal mapping of the plane which is conformal on $\mathbf{C} \backslash \Delta$, where $\Delta$ is a compact set of transfinite diameter 1 , and $f(z)=$ $z+o(1)$ near $\infty$. then

(i) If $f$ is conformal on $E \subset \Delta$ (i.e., $f$ has dilatation $\mu=0$ a.e. on $E$ ),

$$
|f(E)| \leq \pi^{1-1 / K}|E|^{1 / K} .
$$

(ii) If $E \subset \Delta$ with $f$ conformal on $\mathbf{C} \backslash E$, then

$$
|f(E)| \leq K|E| \text {. }
$$

Received by the editors February 7, 1994.

1991 Mathematics Subject Classification. Primary 30C62.

Key words and phrases. Quasiconformal mappings.

Both authors were partially supported by NSF grants. 
(iii) Hence in general for $E \subset \Delta$

$$
|f(E)| \leq K \pi^{1-1 / K}|E|^{1 / K} .
$$

Remarks. Theorem A follows from Theorem 1 via standard distortion estimates for quasiconformal mappings. The constants in Theorem 1 are best possible. Part (ii) is essentially due to Gehring and Reich. Part (i) gives sharp bounds for a conjectured inequality for the singular integral transform

$$
T g(\zeta)=-\frac{1}{\pi} \lim _{\varepsilon \rightarrow 0} \iint_{|z-\zeta|>\varepsilon} \frac{g(z) d x d y}{(z-\zeta)^{2}}
$$

i.e., for every $E \subset \Delta$ we have

$$
\iint_{\Delta \backslash E}\left|T\left(\chi_{E}\right)\right| d x d y \leq|E| \log \frac{\pi}{|E|}
$$

Lemma 1. Let $a_{1}, \ldots, a_{n}$ be positive functions in the unit disk, such that $\log a_{j}$ are harmonic and

$$
\sum_{j=1}^{n} a_{j}(\lambda) \leq 1, \quad|\lambda|<1
$$

Then

$$
\sum_{j=1}^{n} a_{j}(\lambda) \leq\left(\sum_{j=1}^{n} a_{j}(0)\right)^{(1-|\lambda|) /(1+|\lambda|)}, \quad|\lambda|<1 .
$$

The proof is based on the following "Variational Principle" from statistical mechanics which was also used by Astala.

Lemma A. Let $p_{j}>0$ and $q_{j}>0$ be probability distributions on the set $\{1, \ldots, n\}$. Then

$$
-\sum_{j=1}^{n} p_{j} \log q_{j}+\sum_{j=1}^{n} p_{j} \log p_{j} \geq 0
$$

Proof. The left side of the inequality is equal to $\sum q_{j} \phi\left(p_{j} / q_{j}\right)$, where $\phi(x)=$ $x \log x$. This function $\phi$ is convex, so

$$
\sum q_{j} \phi\left(\frac{p_{j}}{q_{j}}\right) \geq \phi\left(\sum q_{j} \frac{p_{j}}{q_{j}}\right)=\phi(1)=0 .
$$

Proof of Lemma 1. For $|\lambda|<1$ and $|z|<1$ define the probability distributions

$$
p_{j}=\frac{a_{j}(\lambda)}{\sum a_{j}(\lambda)} \quad \text { and } \quad q_{j}=\frac{a_{j}(z)}{\sum a_{j}(z)} .
$$

Now fix $\lambda$ and set

$$
H(z)=-\sum p_{j} \log a_{j}(z)+\sum p_{j} \log p_{j}
$$

Observe that $H$ is harmonic in $z$. By Lemma $\mathrm{A}$ and hypothesis (1)

$$
H(z) \geq-\log \sum a_{j}(z) \geq 0 .
$$

Thus by Harnack's inequality

$$
H(z) \geq \frac{1-|z|}{1+|z|} H(0) .
$$


Putting $z=\lambda$ and using Lemma A again we obtain

$$
\begin{aligned}
H(\lambda) & =-\log \sum a_{j}(\lambda) \geq \frac{1-|\lambda|}{1+|\lambda|}\left(-\sum p_{j} \log a_{j}(0)+\sum p_{j} \log p_{j}\right) \\
& \geq \frac{1-|\lambda|}{1+|\lambda|}\left(-\log \sum a_{j}(0)\right),
\end{aligned}
$$

which proves Lemma 1.

Actually we require the continuous version of Lemma 1 . Namely $a(z, \lambda)$ is to be defined on $E \times D$ and $\log a(z, \lambda)$ is harmonic in $\lambda$. If

$$
\iint_{E} a(z, \lambda) d x d y \leq 1, \quad z=x+i y,|\lambda|<1
$$

then we have

$$
\iint_{E} a(z, \lambda) d x d y \leq\left(\iint_{E} a(z, 0) d x d y\right)^{(1-|\lambda|) /(1+|\lambda|)} .
$$

The application to Theorem 1 is immediate. Suppose that $f$ has complex dilatation $\mu$ supported on $\Delta$. Without loss of generality we may assume that $\mu$ is smooth (a uniform bound for the smooth case yields the general uniform bound since the smooth case is dense). Define the function $f_{\lambda} \in \sum\left(K_{\lambda}\right), K_{\lambda}=$ $(1+|\lambda|) /(1-|\lambda|)$, with dilatation

$$
\mu_{\lambda}(z)=\lambda \frac{K+1}{K-1} \mu(z), \quad|\lambda|<1 .
$$

This is done by the standard solution of the Beltrami equation:

$$
f_{\lambda}(z)=z+S \mu_{\lambda}+S \mu_{\lambda} T \mu_{\lambda}+S \mu_{\lambda} T \mu_{\lambda} T \mu_{\lambda}+\cdots,
$$

where $S$ is the complex Cauchy transform. Now $f_{\lambda}$ has Jacobian

$$
J_{\lambda}(z)=\left|\partial_{z} f_{\lambda}(z)\right|^{2}\left(1-\left|\mu_{\lambda}(z)\right|^{2}\right) \text {. }
$$

As the dilatations are smooth this is everywhere nonzero. If $f$ is conformal on $E$ define

$$
a(z, \lambda)=\frac{1}{\pi}\left|\partial_{z} f_{\lambda}(z)\right|^{2}
$$

By the Holomorphic Dependence of Parameter Theorem for the Beltrami equation (see, for example, [5]) $\partial_{z} f_{\lambda}$ is holomorphic in $\lambda$. Thus $\log a(z, \lambda)$ is harmonic for $|\lambda|<1, z \in E$. By the classical Area Theorem for a conformal mapping as $f_{\lambda}(z)=z+o(1), z \rightarrow \infty$,

$$
\iint_{\Delta} J_{\lambda}(z) d x d y \leq \pi
$$

Thus $a(z, \lambda)$ satisfies the continuous version of Lemma 1 giving

$$
\iint_{E} J_{\lambda}(z) \frac{d x d y}{\pi} \leq\left(\frac{|E|}{\pi}\right)^{(1-|\lambda|) /(1+|\lambda|)} .
$$

Setting $\lambda=(K-1) /(K+1)$ gives $\mu_{\lambda}=\mu$ and thus

$$
|f(E)| \leq \pi^{1-1 / K}|E|^{1 / K},
$$

completing the first part of the proof. 
To prove part (ii) and the bound for $T$ we sketch the arguments of Gehring and Reich. This begins with the observation that for any set $G$

$$
\iint_{G}\left|T\left(\chi_{G}\right)\right| d x d y \leq|G|
$$

(by Cauchy-Schwarz as $T$ is a unitary transformation of $L^{2}(\mathbf{C})$ ). Hence for any function $\rho$ supported on $G$ as $T$ is also (almost) self-adjoint

$$
\left|\iint_{G} T(\rho) d x d y\right| \leq\|\rho\|_{\infty}|G| .
$$

Finally for any function $\mu,\|\mu\|_{\infty}=1$, supported on $E$ we define $\mu_{t}(z)=$ $t \mu(z)$ and the corresponding family of normalized maps $f_{t}, 0<t<1, f_{0}(z)=$ $z$ and $f_{|\lambda|}=f$. This can be realised as a deformation family of quasiconformal maps

$$
\begin{gathered}
\frac{\partial f_{t}}{\partial t}=g_{t} \circ f_{t}, \quad g_{t}(z)=z+S \rho_{t}, \\
\rho_{t}=\frac{\mu \circ f_{t}^{-1}}{1-t^{2}\left|\mu \circ f_{t}^{-1}\right|^{2}} e^{2 i \arg \left(\partial_{z} f_{t}^{-1}\right)}, \quad f_{0}(z)=z,
\end{gathered}
$$

by the composition formula for dilatations. Now as $\partial S=T$

$$
\frac{d\left|f_{t}(E)\right|}{d t}=2 \Re \iint_{f_{t}(E)} T\left(\rho_{t}\right) d x d y .
$$

Thus by (2)

$$
\frac{d\left|f_{t}(E)\right|}{d t} \leq 2 \frac{\left|f_{t}(E)\right|}{1-t^{2}}
$$

so by integration

$$
\left|f_{t}(E)\right| \leq \frac{1+t}{1-t}|E|
$$

which proves the result.

The third part follows by writing $f=g \circ h$ where $h$ is conformal on $E$ and $g$ is conformal on $\mathbf{C} \backslash h(E)$. Thus $h$ has dilatation $\mu(z)$ on $\Delta \backslash E$, zero elsewhere, and $g$ has dilatation $\mu\left(h^{-1}(z)\right)$ on $h(E)$, zero elsewhere. We see that $h$ is normalized and so is $g$ as $h(\Delta)$ has transfinite diameter 1 .

The bound on $T$ is also proved by holomorphic deformation. For any function $\mu,\|\mu\|_{\infty}<1$, supported on $\Delta \backslash E$ we define $\mu_{\lambda}(z)=\lambda \mu(z)$ and the corresponding family of normalized maps $f_{\lambda}$. This time we let $\lambda \rightarrow 0$ to find that

$$
\begin{aligned}
\left|f_{\lambda}(E)\right| & =|E|+2 \mathfrak{R}\left(\lambda \iint_{E} T(\mu) d x d y\right)+o(\lambda) \\
& \leq \pi^{2 \lambda+o(\lambda)}|E|^{1-2 \lambda+o(\lambda)}=|E|+2|\lambda||E| \log \frac{\pi}{|E|}+o(\lambda)
\end{aligned}
$$

by part (i) of Theorem 1. Hence we obtain

$$
\left|\iint_{E} T(\mu) d x d y\right| \leq|E| \log \frac{\pi}{|E|}
$$


and so as in the proof of (ii) for all $\mu$ supported on $\Delta \backslash E$ and bounded by 1

$$
\left|\iint_{\Delta \backslash E} T\left(\chi_{E}\right) \bar{\mu}(z) d x d y\right| \leq|E| \log \frac{\pi}{|E|} .
$$

\section{ACKNOWLEDGMENTS}

We thank Kari Astala for sending us a manuscript of his work, David Drasin for inspiring this paper and T. Iwaniec, who urged us to publish this version of the proof.

\section{REFERENCES}

1. K. Astala, Area distortion of quasiconformal mappings, Acta Math. 173 (1994), 37-60.

2. A. Eremenko, On Astala's proof of the area distortion theorem for quasiconformal mappings, preprint, December 1992.

3. F. Gehring and E. Reich, Area distortion under quasiconformal mappings, Ann. Acad. Sci. Fenn. Ser. AI 388 (1966), 1-14.

4. D. Hamilton, Area distortion of quasiconformal mappings, preprint, December 1992.

5. O. Lehto and K. Virtanen, Quasiconformal mappings in the plane, Springer-Verlag, Berlin and New York, 1973.

Department of Mathematics, Purdue University, West Lafayette, Indiana 47907

E-mail address: eremenko@math.purdue.edu

Department of Mathematics, University of Maryland, College Park, Maryland 20742 